\title{
MicroRNA 196a-2 Polymorphism and Serum Tyrosinase Level in Vitiligo Patients
}

\author{
K.M.Hussein ${ }^{1}$, I.Y.Abd Allah ${ }^{1}$, H.H.Sabry ${ }^{1}$, A.A.Elfalah ${ }^{2}$ and R.A.Abd Elsamie ${ }^{1}$ \\ ${ }^{1}$ Dermatology \& Andrology Dept., Faculty of Medicine, Benha Univ., Benha ,Egypt \\ ${ }^{2}$ Clinical and Chemical Pathology Dept., Faculty of Medicine, Benha Univ.. Benha, Egypt \\ E-Mail: dr_romia@yahoo.com
}

\begin{abstract}
MicroRNAs (miRNAs) are the key regulators of a variety of biological processes such as cell proliferation, apoptosis and tumorigenesis. It was also demonstrated to target tyrosinase, which plays an important role in melanin synthesis. The aim of this work was to evaluate microRNA 196a-2 polymorphism and serum tyrosinase levels in vitiligo patients. The current study was a case-control study that was conducted on 100 participants. Fifty of them were patients with vitiligo, and they were located in group 1. Group 2 contained 50 age and sex matched healthy control participants. Approval of the Local Ethics Committee on Research involving human subjects of Benha Faculty of Medicine and written informed consents of participants were obtained before samples collection. Serum tyrosinase level and microRNA 196a-2 polymorphism were evaluated in all participants. The level of serum tyrosinase is significantly higher in patients with vitiligo when compared to the control subjects. T allele and TT genotype of miRNA 196a-2 polymorphism were found to have a significant relation to the development of vitiligo. MiRNA 196a-2 TT genotype and the high serum tyrosinase levels may play a role in vitiligo pathogenesis.
\end{abstract}

Keywords: Vitiligo,Tyrosinase, Polymorphism, microRNA.

\section{Introduction}

Vitiligo is an idiopathic skin disease characterized by selective destruction of melanocytes leading to depigmentation in the form of milky white macules or patches. Vitiligo occurs worldwide with an estimated prevalence of $0.5-1 \%$. In almost half of the patients, vitiligo starts before the age of 20 years, however, it can be seen at any age group with no significant sex difference [1].

Although the etiopathogenesis of vitiligo is not yet fully understood, the autoimmune, auto cytotoxic, neural, and biochemical-based hypotheses are considered. Genetic predisposition and triggering factors have roles in the emergence of the disease [2].

Micro-RNAs contribute to the cellular regulatory processes via their capacity to alter the expression of approximately $60 \%$ human genes at both post-transcription and translation levels. Therefore, miRNAs are of great importance in diverse physiological and developmental processes in humans including development and function of melanocytes as well as immune cells [3].

Recently, miRNA-196-a2 gained a lot of attention. It has been reported to be deregulated in various cancer types and consequently, this up- or down-regulation may impact tumor malignancy or drug resistance according to the downstream target genes it affects. Bioinformatics analysis had shown that miR-196-a2 could target many genes enriched in cell cycle regulation, survival and apoptosis [4].

It was found that miR-196a2 polymorphism contributes to pathogenesis of variety of diseases and is possible genetic predisposing factor. MiRNA196a2 was found to be associated with many systemic diseases such as coronary artery disease [5], GI cancers [4], increase the risk of ischemic stroke [6] as well as certain types of HCC [7].

However, little is known regarding the contribution of genetic variations in miRNAs to the development of vitiligo [8]. It was hypothesized that SNPs in miR-196a-2 could potentially alter regulation of the expression of the target TYRP1, an enzyme that may use an oxidative inducer as a substrate and promote ROS formation by catalyzing quinone production in melanocytes, leading to individual susceptibility to vitiligo [9].

In addition, it was also demonstrated that miR-196a-2 targets Tyrp1gene. And the rs $11614913 \mathrm{~T} / \mathrm{C}$ change in miRNA196a-2 could down-regulate the cellular level of ROS and protect human melanocytes from apoptosis by suppressing the expression of Tyrp1 [10].

\section{Subjects and methods 2.1Methodology}

The current study was a case-control study that was conducted on patients with vitiligo in Benha University Hospital. This study was conducted on 100 participants. Fifty of them were patients with vitiligo, and they were located in group 1. Group 2 contained 50 control participants matching to the patients in age and sex. Approval of the Local Ethics Committee on Research involving human subjects of Benha Faculty of Medicine and written informed consents of participants were obtained before samples collection.

In group 1, the patients assessed with full history taking, general and dermatological examination with photographic documentation. The severity of the condition was assessed by rule of nine and VASI score. Then blood samples were taken for laboratory investigations. Determination of the miRNA 196a-2 polymorphism with 
PCR as well as assay of the serum level of tyrosinase enzyme using ELISA were done in all participants.

\subsection{Statistical analysis}

Qualitative data were summarized in the form of frequency and percentage. Mean and SD were obtained for quantitative data, while categorical data were presented by number and percentage. One way analysis of variance (ANOVA) test, paired and independent sample t-tests were used for comparing means between groups. Tests used for association were Chi square (X2) or Mont-Carlo Exact test (MCET). P -value was adopted to be $<0.05$.

\section{Results and discussion}

The patients and control groups were matching regarding age $(37.14 \pm 15.6$ versus $35.2 \pm 9.8, \mathrm{p}=0.56)$ and gender (30\% male patients for both groups, $\mathrm{p}=1)$. The clinical findings of patients are shown in Table(1).

Table (1) Vitiligo characteristics among studied patients $(\mathrm{n} .=50)$.

\begin{tabular}{lcc}
\hline TOTAL & $\mathbf{n}$ & $\mathbf{\%}$ \\
& $\mathbf{5 0}$ & $\mathbf{1 0 0}$ \\
\hline Type of vitiligo & 38 & 76 \\
Vulgaris & 6 & 12 \\
Acral & 5 & 10 \\
Acrofacial & 1 & 2 \\
Focal & & \\
Associated autoimmune diseases & 41 & 82 \\
No & 3 & 6 \\
Rheumatoid arthritis & 2 & 4 \\
Thyroiditis & 2 & 4 \\
Grave's disease & 2 & 4 \\
Alopecia areata & & \\
Previous lines of treatment & 23 & 46 \\
Phototherapy only & 7 & 14 \\
Topical only & 12 & 24 \\
Phototherapy + topical & 3 & 6 \\
Phototherapy + topical + & \\
antioxidants & & \\
No previous treatment & 5 & 10 \\
Surface area of vitiligo & & \\
< 10\% & 10 & 20 \\
10 - <20 & 21 & 42 \\
$\mathbf{2 0}$ - <30 & 9 & 18 \\
\hline
\end{tabular}

\begin{tabular}{lcc}
\hline$\geq 30$ & 10 & 20 \\
VASI score $($ mean \pm SD) & $\mathbf{9 . 6 \pm 6 . 8}$ & \\
\hline
\end{tabular}

Serum tyrosinase level was found to be higher in group $1(89.05 \pm 2.6)$ than in group $2(80.4 \pm 1.34)$. There was a statistically highly significant difference between the 2 groups ( $\mathrm{P}<0.0001)$ Fig (1).

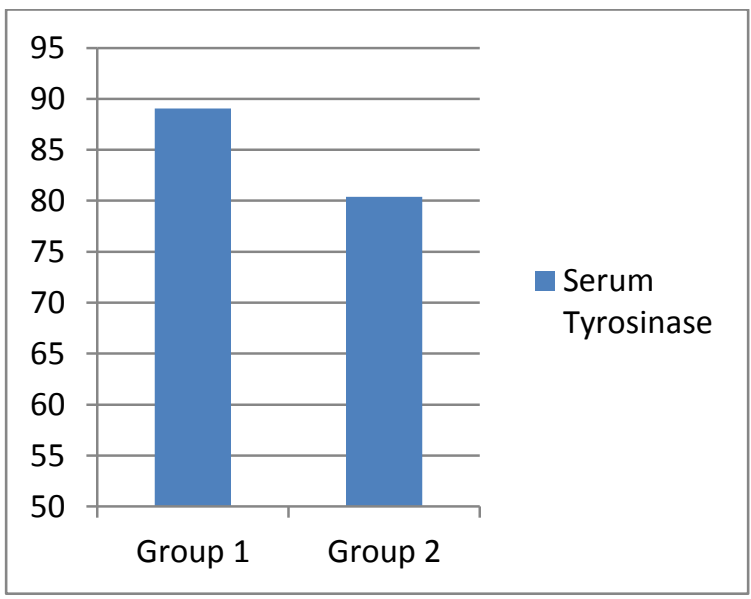

Fig (1) The mean serum tyrosinase level among studied groups.

Regarding the miRNA 196-a2 polymorphism, it was found that TT genotype was more prevalent among the diseased patients, while CC genotype was more prevalent among the control group Table (2).

Micro-RNAs contribute to the cellular regulatory processes via their capacity to alter the expression of approximately $60 \%$ human genes at both post-transcription and translation levels.

Therefore, miRNAs are of great importance in diverse physiological and developmental processes in humans including the development and function of melanocytes as well as immune cells [3].

Recently, miRNA-196-a2 gained a lot of attention. It has been reported to be deregulated in various cancer types and consequently, this up- or down-regulation may impact tumor malignancy or drug resistance according to the downstream target genes it affects. Bioinformatics analysis had shown that miR-196-a2 could target many genes enriched in cell cycle regulation, survival, and apoptosis [4].

Table (2) MiRNA 196a2 polymorphism between cases and controls groups.

\begin{tabular}{cccccc}
\hline $\begin{array}{c}\text { MiRNA 196a2 } \\
\text { polymorphism }\end{array}$ & \multicolumn{2}{c}{ Group 1 } & \multicolumn{2}{c}{ Group 2 } & \multirow{2}{*}{ P value } \\
\cline { 2 - 5 } & $\mathbf{n}$ & $\mathbf{\%}$ & $\mathbf{n}$ & $\mathbf{\%}$ & \\
\hline CC & 21 & 42 & 31 & 62 & MCET $=15.2$ \\
\hline CT & 0 & 0 & 2 & 4 & $\mathrm{P}=0.014^{*}$ \\
\hline
\end{tabular}




\begin{tabular}{ccccc}
\hline TT & 29 & 58 & 17 & 34 \\
\hline Total & 50 & 100 & 50 & 100 \\
\hline
\end{tabular}

No significant relationship has been detected between the miRNA 196-a2 genotypes or the serum tyrosinase levels with any of the study variables Tables $(3,4)$.

Table (3) Relationship between miRNA 196-a2 polymorphism and study variables.

\begin{tabular}{|c|c|c|c|c|c|}
\hline & \multicolumn{4}{|c|}{ MiRNA 196 a2 polymorphism } & \multirow{4}{*}{$P$ value } \\
\hline & \multicolumn{2}{|c|}{$\mathbf{C C}$} & \multicolumn{2}{|c|}{ TT } & \\
\hline & $\mathbf{n}$ & $\%$ & $\mathbf{n}$ & $\%$ & \\
\hline Total & 21 & 100 & 29 & 100 & \\
\hline Associated autoimmune disease & & & & & \multirow{6}{*}{$\begin{array}{c}\mathrm{MCET}=4.11 \\
\mathrm{P}=0.39\end{array}$} \\
\hline No & 15 & 71.4 & 26 & 89.7 & \\
\hline Rheumatoid arthritis & 2 & 9.5 & 1 & 3.4 & \\
\hline Thyroiditis & 1 & 4.8 & 1 & 3.4 & \\
\hline Grave's disease & 2 & 9.5 & 0 & 0 & \\
\hline Alopecia areata & 1 & 4.8 & 1 & 3.4 & \\
\hline \multicolumn{6}{|l|}{ Family History } \\
\hline Negative & 15 & 71.4 & 17 & 58.6 & \multirow{2}{*}{$\begin{array}{c}X 2=0.87 \\
P=0.35\end{array}$} \\
\hline Positive & 6 & 28.6 & 12 & 41.4 & \\
\hline \multicolumn{6}{|l|}{ Type of vitiligo } \\
\hline Vulgaris & 15 & 71.4 & 23 & 79.3 & \multirow{4}{*}{$\begin{array}{c}\mathrm{MCET}=1.64 \\
\mathrm{P}=0.6\end{array}$} \\
\hline Acral & 3 & 14.3 & 3 & 10.3 & \\
\hline Acrofacial & 3 & 14.3 & 2 & 6.9 & \\
\hline Focal & 0 & 0 & 1 & 3.4 & \\
\hline \multicolumn{6}{|l|}{ Course of the disease } \\
\hline Progressive & 10 & 47.6 & 12 & 41.4 & \multirow{4}{*}{$\begin{array}{c}\mathrm{X} 2=1.15 \\
\mathrm{P}=0.16\end{array}$} \\
\hline Intermittent & 4 & 19 & 10 & 34.5 & \\
\hline Stationary & 4 & 19 & 4 & 13.8 & \\
\hline Regressive & 3 & 14.3 & 3 & 10.3 & \\
\hline
\end{tabular}

Table (4) Relationship between s.Tyrosinase and study variables

\begin{tabular}{lccc}
\hline & \multicolumn{3}{c}{ S. Tyrosinase level (mg/dl) } \\
\cline { 2 - 4 } Gender & Mean \pm SD & F & P value \\
Male & $88.89 \pm 3.28$ & 0.133 & 0.7 \\
Female & $89.14 \pm 2.3$ & & \\
\hline Age & & & \\
$<\mathbf{2 0}$ & $89.87 \pm 2.46$ & & 0.7 \\
$\mathbf{2 0 - 2 9}$ & $89.4 \pm 2.9$ & 0.401 & \\
$\mathbf{3 0 - 3 9}$ & $88.49 \pm 2.5$ & & \\
$\geq \mathbf{4 0}$ & $89 \pm 2.7$ & & \\
\hline Type & & & \\
Vulgaris & $89.3 \pm 2.4$ & & \\
Acral & $87.3 \pm 3.7$ & \multirow{2}{*}{1.02} & \\
Acrofacial & $88.9 \pm 2.4$ & & \\
Focal & 89.7 & & \\
\hline
\end{tabular}

However, little is known regarding the contribution of genetic variations in miRNAs to the development of vitiligo [8]. It was hypothesized that SNPs in miR-196a-2 could potentially alter the regulation of the expression of 
the target TYRP1, an enzyme that may use an oxidative inducer as a substrate and promote ROS formation by catalyzing quinone production in melanocytes, leading to individual susceptibility to vitiligo [9].

In addition, Cui et al. [10], also demonstrated that miR196a-2 targets Tyrp1gene, and the rs $11614913 \mathrm{~T} / \mathrm{C}$ change in miRNA196a-2 could down-regulate the cellular level of ROS and protect human melanocytes from apoptosis by suppressing the expression of Tyrp1.

The aim of this work was to evaluate microRNA 196a2 polymorphism and serum tyrosinase levels in vitiligo patients.

Vitiligo could be considered as a polygenetic disease; however, the genetic risk is not absolute. So, positive family history is one of the strong associations with vitiligo. Mohammed et al. [11], found that the range of family history in vitiligo varied from $6.25 \%$ up to $38 \%$ in some studies. And this is in agreement with the current study results that demonstrated that positive family history was $36 \%$. And this percentage is higher than that was concluded by Butt et al. [12], which was $22 \%$. The patients were considered to have a family history if they had one or more first- to third-degree relatives with this condition.

Vitiligo is widely accepted to be an autoimmune disease and commonly associated with other autoimmune diseases. In this study, it was found that about $18 \%$ of the patients suffered also from other associated autoimmune diseases. This is comparable to that was found by Cui et al. [10], who mentioned that the incidence was $13 \%$. However, this percentage was less than that mentioned by Huang et al. [8], as it was $26 \%$ in his study. This may be due to the exclusion of diabetes mellitus from the list of autoimmune disease in the current study. The exclusion of diabetes from the autoimmune diseases was due to the fact that the autoimmune mechanism plays a minor role in the pathogenesis of this disease especially type II, as the genetic, ethnic, environmental as well as nutritional factors play the main pathogenic roles [13].

Regarding the level of serum tyrosinase level, it was found that there was a highly significant relation between the higher levels of serum tyrosinase enzyme and the development of vitiligo. The mean levels were $89.05 \mathrm{pg} / \mathrm{ml}$ and $80.4 \mathrm{pg} / \mathrm{ml}$ in group 1 and 2 respectively. This finding is similar to that concluded by Cui et al. [10], who reported $95 \mathrm{pg} / \mathrm{ml}$ and $80 \mathrm{pg} / \mathrm{ml}$ for the case and control group respectively.

Serum tyrosinase level had no relation to gender. This is in agreement with Cui et al. [10]. They mentioned also that the level is increasing with age, but the current study showed that there was no relation between the level of serum tyrosinase and the age of the patient.

T allele of miRNA 196a-2 polymorphism was found to have a significant relation with the development of vitiligo, as it was more prevalent in group 1 . The same was stated by Huang et al. [8[, who also found that incidence of vitiligo development was high with TT genotype and low with CC genotype of the same gene.

Although this study showed no statistically significant relation with the presence of associated autoimmune disease or with positive family history, the opposite was concluded by Huang et al. [8], who found that CC genotype is associated with less association with other autoimmune disease and also less incidence of positive family history.

MiRNA-196a-2 C allele reduced the protein level of Tyr in PIG1 cells by inhibiting the expression of Tyrp1 [10]. Also, the experimental data showed that upregulation of miR-196a-2 expression decreased the protein levels of TYRP1, whereas inhibition of miR-196a-2 expression increased the protein levels [8]. These facts are completely in agreement with the current results.

\section{Conclusion}

MiRNA 196a-2 TT genotype and the high serum tyrosinase levels may play a role in vitiligo pathogenesis.

\section{References}

[1] K. Boniface, J. Seneschal, M. Picardo and A. Taïeb: Vitiligo: focus on clinical aspects, immunopathogenesis, and therapy. Clin Rev Allergy Immunol; vol. 54, pp.52-67, 2018.

[2] G. Iannella, A. Greco, D. Didona, B. Didona, G. Granata, A. Manno, B. Pasquariello and G. Magliulo: Vitiligo: Pathogenesis, clinical variants and treatment approaches. Autoimmun Rev; vol. 15, pp.335-343, 2016.

[3] M. Mansuri, M. Singh and R. Begum: miRNA signatures and transcriptional regulation of their target genes in vitiligo. J Dermatol Sci; vol. 6, pp.84: 50-, 2014.

[4] M. Fawzy, E. Toraih, A. Ibrahiem, H. Abdeldayem, A. Mohamed and M. Abdel-Daim: Evaluation of miRNA196a 2 and apoptosis related target genes: ANXA1, DFFA and PDCD4 expression in gastrointestinal cancer patients: A pilot study. PLoS ONE; 12(11): e0187310, 2017.

[5] M. Buraczynska, P. Zukowski, P. Wacinski, K. Ksiazek and W. Zaluska: Polymorphism in microRNA$196 \mathrm{a} 2$ contributes to the risk of cardiovascular disease in type 2 diabetes patients. J Diabetes Complications; vol. 28, pp. 617-620, 2014.

[6] K.. Zhu, H. Hou ,L. Ma,Z. Yang and L. Pan: Associations of miR-146a, miR-149, miR-196a2, and miR-499 Polymorphisms with Ischemic Stroke in the Northern Chinese Han Population. Med Sci Monit, vol. 24, pp. 7366-7374, 2018.

[7] W. Hou, Q. Tian, J. Zheng and H. Bonkovsky: MicroRNA-196 represses bach1 protein and HCV gene expression in human hepatoma cells expressing 
hepatitis C viral proteins. Hepatology; vol. 51, pp. 1494-1504, 2010.

[8] Y. Huang, X. Yi, Z. Jian, C. Wei, S. Li, C. Cai, P. Zhang, K. Li, S. Guo, L. Liu, Q. Shi, T. Gao and C. Li: A single-nucleotide polymorphism of miR-196a-2 and vitiligo: an association study and functional analysis in a Han Chinese population. Pigment Cell Melanoma Res; vol. 26, pp. 338-347, 2012.

[9] P. Manga, D. Sheyn, F. Yang, R. Sarangarajan and R. Boissy: A role for tyrosinase-related protein 1 in 4-tertbutylphenol-induced toxicity in melanocytes. Am J Pathol; vol. 169, pp. 1652:62, 2006.

[10] T. Cui, X. Yi, W. Zhang, C. Wei, F. Zhou, Z. Jian, G. Wang, T. Gao, Y. Li and K. Li: miR-196a-2 rs11614913 polymorphism is associated with vitiligo by affecting heterodimeric molecular complexes of Tyr and Tyrp1. Arch Dermatol; vol. 307, pp. 683-692, 2015.

[11] G. Mohammed, A. Gomaa and M. Al-Dhubaibi: Highlights in pathogenesis of vitiligo. World J Clin Cases; vol. 3, pp. 221-230, 2015.

[12] G. Butt, F. Altaf, U. Wazir and S. Pal: Familial frequency of vitiligo and its association with autoimmune disorders. J Pakistan Association Dermatol; vol. 15, pp.101-104, 2015.

[13] E. Adeghate, P. Schattner and E. Dunn: An update on the etiology and epidemiology of diabetes mellitus. Ann NY acad sci;vol. 1084, pp. 1-29, 2006. 